\title{
An unusual cause of haemorrhagic shock from a subcutaneous haematoma: a Morel-Lavallée lesion
}

\author{
Renhao Desmond Mao ${ }^{1}$, MCEM, MMed, Enjiu Pauleon Tan², MrCs, Hsin Kai Goh ${ }^{1}$, Mrcs, fams
}

\begin{abstract}
A 20-year-old man presented to our emergency department after he was hit by a forklift. He developed haemorrhagic shock from a subcutaneous haematoma in his left thigh and required monitoring in the surgical intensive care unit. He stabilised with aggressive fluid resuscitation with crystalloids and blood transfusion. The recovery was complicated by an infection of the subcutaneous haematoma. Following open drainage of the infected subcutaneous haematoma, he improved and was discharged. To the best of our knowledge, this is the first reported case of a subcutaneous haematoma causing haemorrhagic shock.
\end{abstract}

Keywords: emergency, haemorrhagic shock, Morel-Lavallée lesion, subcutaneous haematoma

\section{INTRODUCTION}

The Morel-Lavallée lesion is a rare condition, first described by French physician Maurice Morel-Lavallée in 1853. ${ }^{(1)}$ The condition, which is due to a closed, internal degloving injury usually resulting from run-over accidents, commonly occurs over the greater trochanter but may also occur in the flank and lumbosacral region. ${ }^{(1-3)}$ The result of this lesion is bleeding into the subcutaneous tissue plane, which then causes the development of a haematoma. We herein report the unusual occurrence of a Morel-Lavallée lesion presenting with haemorrhagic shock in a 20-year-old man following a worksite accident. To the best of our knowledge, this is the first reported case of a Morel-Lavallée lesion causing haemorrhagic shock.

\section{CASE REPORT}

A previously healthy 20-year-old Bangladeshi man was hit by a reversing forklift at work. At the time of presentation to the emergency department (ED), he was hypertensive (blood pressure [BP]: 166/76 mmHg) and tachycardic (heart rate [HR]: 149 bpm). He appeared confused and could only make groaning noises. His external injuries included multiple facial lacerations and a deformity over the distal shin. There was no evidence of any other external injury. Radiological examination confirmed a closed fracture of the left tibia.

Five minutes following arrival to the $\mathrm{ED}$, the patient became hypotensive (BP: 70/40 $\mathrm{mmHg}$ ) and remained tachycardic (HR: $134 \mathrm{bpm}$ ). In view of the decreased consciousness level and the clinical findings, which were suggestive of shock, he was intubated for airway protection. Fluid resuscitation had been initiated on his arrival and two units of packed red blood cells, $1.0 \mathrm{~L}$ of saline, 1.5 L of Hartmann's solution and $500 \mathrm{~mL}$ of Gelafundin were administered.

Focused assessment with sonography for trauma examination was negative for intra-abdominal free fluid. The patient's initial serum lactate was $7.9 \mathrm{mmol} / \mathrm{L}$ and haemoglobin level was $10.9 \mathrm{~g} / \mathrm{dL}$. Computed tomography (CT) of the brain, cervical spine, thorax, abdomen and pelvis were performed. The only significant abnormality detected was a subcutaneous haematoma in the left lateral hip region extending into the pelvis (Fig. 1). No contrast extravasation, evidence of intracranial bleed, or any intrathoracic and intra-abdominal injury was seen. There was also no free fluid in the abdomen or pelvis, and no evidence of cardiac tamponade or pneumothorax.

Following the resuscitation efforts, the patient's BP improved to $99 / 53 \mathrm{mmHg}$. For further management, he was sent to the surgical intensive care unit (ICU), where his BP was stable although he remained persistently tachycardic. At six hours after presentation to the $E D$, the rechecked haemoglobin level was $5.1 \mathrm{~g} / \mathrm{dL}$. No increased swelling was noted at his fracture site (i.e. left closed tibia). However, he again became hypotensive and required inotropic support with intravenous noradrenaline. He developed increasing areas of ecchymosis and an expanding swelling over his left flank and thigh. His left thigh and lower limb were wrapped in a compressive bandage in an attempt to minimise swelling. Repeat mesenteric CT angiography of the abdomen and pelvis showed a large subcutaneous haematoma extending from the left flank to the left lateral and bilateral anterior pelvic walls (Figs. $2 \& 3$ ). There was no evidence of active contrast extravasation. Further resuscitation with blood products and fluids was successful in gradually reversing the patient's tachycardia after 72 hours in the ICU. During that time, another 2.0 L of Hartmann's solution, $1.0 \mathrm{~L}$ of Voluven, ten units of packed red blood cells, 1.0 L of fresh frozen plasma and four units of platelets were administered. The patient's left tibia fracture was minimally displaced and treated nonoperatively. A left above-knee backslab was applied to immobilise the fracture site.

The patient developed fever as an inpatient on Day 6 of admission. Clinically, the fever was attributed to infection of the left-sided subcutaneous haematoma, for which he subsequently underwent surgical drainage. Intraoperatively, $1.4 \mathrm{~L}$ of haematoma was drained from his left thigh. Wound cultures of the haematoma grew Escherichia coli, Stenotrophomonas maltophilia

${ }_{1}^{1}$ Department of Acute and Emergency Care, ${ }^{2}$ Department of General Surgery, Khoo Teck Puat Hospital, Singapore

Correspondence: Dr Mao Renhao Desmond, Registrar, Department of Acute and Emergency Care, Khoo Teck Puat Hospital, 90 Yishun Central, Singapore 768828. mao.desmond.r@alexandrahealth.com.sg 


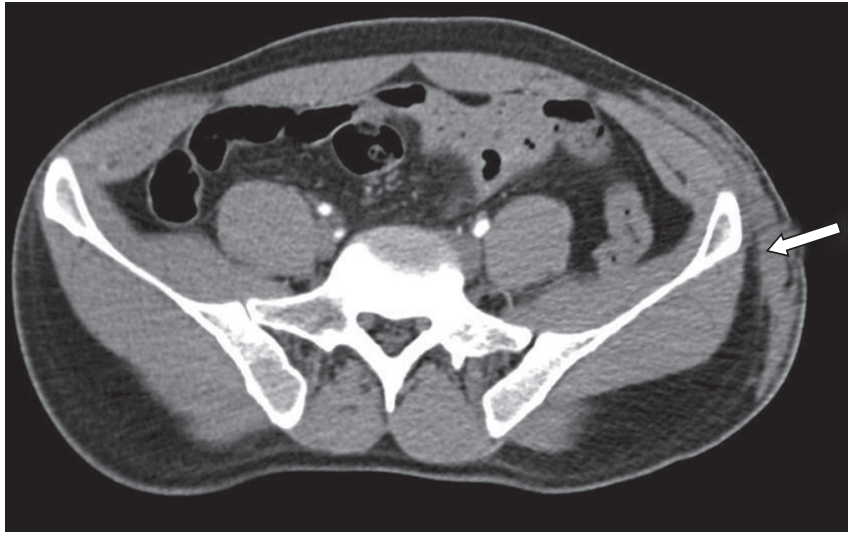

Fig. 1 Initial CT angiogram done on arrival to the emergency department shows a subcutaneous haematoma around the left iliac crest (arrow).

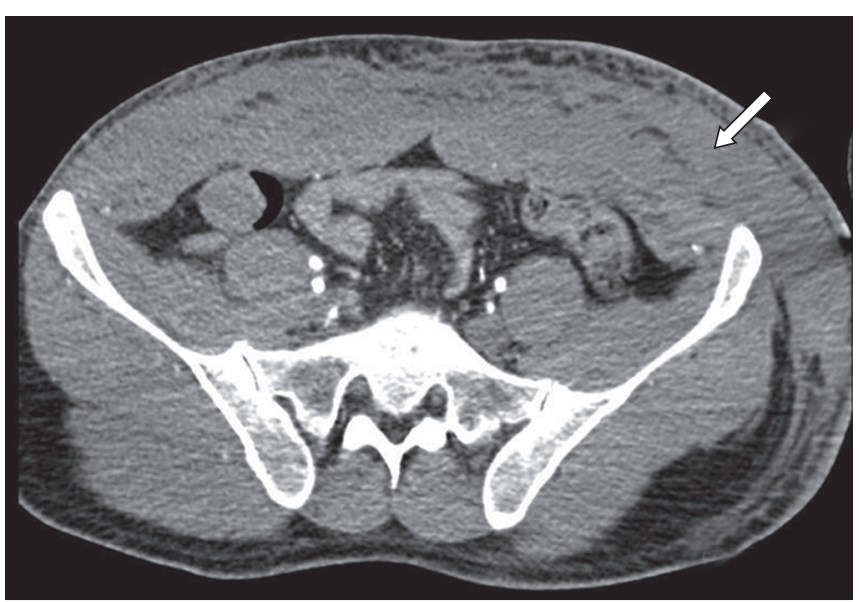

Fig. 2 Transverse mesenteric CT angiogram done 24 hours after presentation to the emergency department shows an increase in the size of the subcutaneous haematoma (arrow).

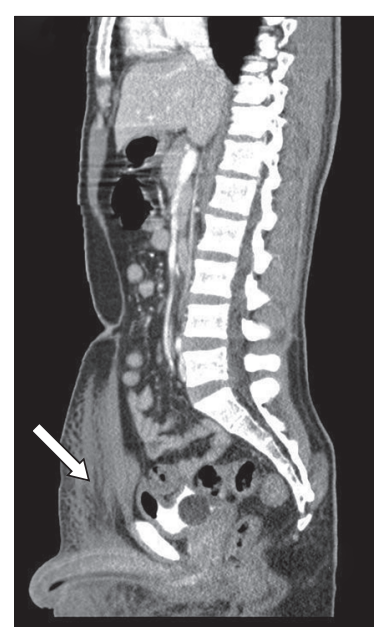

Fig. 3 Sagittal mesenteric CT angiogram done 24 hours after presentation to the emergency department shows an increase in the size of the subcutaneous haematoma (arrow).

and Enterobacter. Following a further ten days of antibiotics, the post-drainage wound defect was covered with a split-skin graft. The patient's fever resolved and he was discharged to a step-down care facility shortly after. At review six months after the accident, the patient was fully ambulant and the surgical wound on his left thigh had healed completely.

\section{DISCUSSION}

A history of trauma, coupled with findings of tachycardia, hypotension, confusion and a raised lactate level, suggests that our patient was in at least Class III shock. ${ }^{(4)}$ There was no obvious external bleeding. Clinical examination and radiological workup had ruled out tension pneumothorax, haemothorax, cardiac tamponade and intra-abdominal haemorrhage as causes of shock in our patient. He had a single long bone fracture of the tibia, which was not actively expanding in size. This injury in itself was deemed insufficient to cause Class III shock. ${ }^{(4)}$ In the setting of trauma, the most likely cause of the state of shock, despite the absence of an obvious cause, is unrecognised haemorrhage. Our patient's partial response to the administration of fluids and blood suggests ongoing blood loss or inadequate resuscitation. ${ }^{(4)}$

The Morel-Lavallée lesion is a closed, internal degloving injury that may occur in association with pelvic and acetabular fractures, but it may also occur in the absence of fractures. ${ }^{(1,2)}$ Essentially, shearing forces result in the separation of the hypodermis from the underlying fascia. This potential space is then filled with blood and other necrotic tissue, and the collection is referred to as a Morel-Lavallée lesion. The lesion may spontaneously resolve, or become encapsulated and persistent.

In the present case, careful interpretation of the initial CT revealed a Morel-Lavallée lesion in the left flank. The lesion spanned over a large surface area and was fairly subtle on the initial CT. The lack of an early cutaneous sign is a factor that could mislead the treating physician from suspecting the presence of the lesion. In a review of 16 cases, Hudson et al reported that the diagnosis of Morel-Lavellée lesion was initially missed in one-third of the cases.(2) If not specifically sought for, subcutaneous haemorrhage may go unrecognised for long periods with devastating consequences. This case report shows that the traumatologist should not underestimate subcutaneous haemorrhage as a potential cause of haemorrhagic shock. If in doubt, the treating physician must be prepared to obtain repeat CT. In the present case, repeat CT showed expansion of the subcutaneous haematoma, which is confirmed to be the source of continued bleeding. To our knowledge, this is the first reported case of a closed, internal degloving injury that caused haemorrhagic shock.

Despite being a closed injury, other potential complications of a Morel-Lavallée lesion include wound dehiscence and deep bone and soft tissue infections. The literature describes different methods to treat this injury. ${ }^{(1-3,5-8)}$ While some authors suggest open surgical debridement, ${ }^{(2,3,5)}$ others have described alternative forms of treatment such as compressive bandaging, ${ }^{(6,7)}$ percutaneous drainage, ${ }^{(6,7)}$ and injection of sclerosing agents. ${ }^{(8)}$ Lin et $\mathrm{al}^{(6)}$ successfully treated a patient with echo-guided percutaneous aspiration coupled with the application of a compressive bandage over the haematoma site. In our patient, expectant management with compressive bandaging was adequate in controlling the initial expansion of the haematoma. However, the development of an infected haematoma eventually required formal surgical drainage and subsequent skin grafting. 
In conclusion, this case report served to highlight two complications of the Morel-Lavallée lesion. First, the lesion may be an unrecognised cause of haemorrhagic shock, and second, subsequent infection of the lesion may occur. Therefore, the attending physician should be aware of the possibility of a Morel-Lavallée lesion and monitor the development of any complications if the initial treatment is conservative.

\section{REFERENCES}

1. Vanhegan IS, Dala-Ali B, Verhelst L, Mallucci P, Haddad FS. The morellavallée lesion as a rare differential diagnosis for recalcitrant bursitis of the knee: case report and literature review. Case Rep Orthop 2012; 2012:593193.

2. Hudson DA, Knottenbelt JD, Krige JE. Closed degloving injuries: results following conservative surgery. Plast Reconstr Surg 1992; 89:853-5

3. Hak DJ, Olson SA, Matta JM. Diagnosis and management of closed internal degloving injuries associated with pelvic and acetabular fractures: the Morel-Lavallée lesion. J Trauma 1997; 42:1046-51.

4. American College of Surgeons Committee on Trauma. Advanced Trauma Life Support巴 Student Course. 8th ed. Chicago: American College of Surgeons, 2008.

5. Asprinio DE, Helfet DL, Tile M. Complications. In: Tile M, eds. Fractures of the pelvis and acetabulum. 2nd ed. Baltimore, MD: Williams and Wilkins, 1995: 451-67.

6. Lin HL, Lee WC, Kuo LC, Chen CW. Closed internal degloving injury with conservative treatment. Am J Emerg Med 2008; 26:254.e5-6.

7. Letournel E, Judet R. Fractures of the acetabulum. 2nd ed. Berlin: SpringerVerlag, 1993.

8. Tejwani SG, Cohen SB, Bradly JP. Management of Morel-Lavallee lesion of the knee: twenty-seven cases in the national football league. Am J Sports Med 2007; 35:1162-7. 